Citation: Kragh H. (2021) Review of What Is a Chemical Element? By Eric Scerri and Elena Ghibaudi. Substantia 5(1): 157-158. doi: 10.36253/Substantia-1184

Copyright: (c) 2021 Kragh H.. This is an open access, peer-reviewed article published by Firenze University Press (http://www.fupress.com/substantia) and distributed under the terms of the Creative Commons Attribution License, which permits unrestricted use, distribution, and reproduction in any medium, provided the original author and source are credited.

Data Availability Statement: All relevant data are within the paper and its Supporting Information files.

Competing Interests: The Author(s) declare(s) no conflict of interest.
Book Reviews

\section{Review of What Is a Chemical Element? By Eric Scerri and Elena Ghibaudi, eds. Oxford: Oxford University Press, 2020}

\author{
Helge Kragh \\ Niels Bohr Institute, University of Copenhagen, Denmark \\ E-mail: helge.kragh@nbi.ku.dk
}

Whereas philosophy of physics and philosophy of biology have for long been well-established academic sub-disciplines, philosophy of chemistry in its modern sense is of more recent origin. The field essentially originated in the late 1980s, not least through the pioneering works and organizational efforts of Eric Scerri. Since then philosophy of chemistry has flourished and attracted much attention not only from historians and philosophers of science but also from some practicing chemists. As indicated by its title, the present work edited by Scerri and Elena Ghibaudi focuses on the nature and meaning of a chemical element, obviously a concept at the very heart of the chemical sciences. The book contains 14 chapters written by specialists in the philosophy and foundation of chemistry, with all of the chapters expertly discussing aspects of what constitutes an element and how the concept has developed through history. Some of the chapters are historically oriented, examining the development from Lavoisier over Dalton to Mendeleev and further on. This is the topic of a penetrating essay by Bernadette BensaudeVincent, whereas Nathan Brooks focuses on Mendeleev's ideas prior to his 1869 formulation of the periodic system. Contrary to the common conception, Brooks argues that Mendeleev was not opposed to the idea of elements made up of subatomic units.

Other contributions are primarily of a conceptual and philosophical nature, and others again relate to the history of ideas, such as do the contributions of Farzad Mahootian and Klaus Ruthenberg. While Mahootian discusses the influence of Immanuel Kant and Ernst Cassirer, Ruthenberg calls attention to the non-atomistic ideas of František Wald, Wilhelm Ostwald and Gaston Bachelard. The latter, a French philosopher, introduced the notion of "metachemistry," which to him had quite different connotations than metaphysics. Incidentally, the term is also occasionally used by historians of science to characterize speculative trends in late-nineteenth-century chemistry, such as the views of William Crookes.

A common theme in many of the chapters is the current official definition of an element as given by IUPAC, which defines the concept in a some- 
what ambiguous dual form. On the one hand there is the abstract meaning of a species of atoms as given by the number of protons in the nucleus (the atomic number); and on the other hand there is an operational meaning stated in terms of the macroscopic concept of a pure substance. The two meanings are meant to coexist, but do they? As Sarah Nijmans and other authors point out, the IUPAC definition is problematic for both historical and philosophical reasons. While most of the authors are highly critical to the IUPAC formulation, Mahootian suggests that its two parts are not contradictory but rather stand in a complementary relationship. He argues that Niels Bohr's famous but vague principle of complementarity can and should be extended from the realm of quantum physics to elucidate also problems in the philosophy of chemistry. IUPAC's definition of an element reflects to some extent the important ideas of Fritz Paneth, the Austrian radiochemist who in influential works between 1916 and 1962 analyzed in depth the concept of an element. Several of the contributions deal with Paneth's ideas and their later impact, a topic discussed in some detail by Scerri, Hijmans and Joseph Earley, among others.

One of the essays, written by Ghibaudi, Alberto Regis and Ezio Roletto, includes a discussion of the chemical element from an educational perspective, albeit in a rather abstract way which may not be directly relevant to teachers of chemistry. In an interesting chapter on "The Existence of Elements and the Elements of Existence" Robin Hendry focuses on the chemical element from an ontological point of view and with an emphasis on the artificially produced elements at the end of the periodic table. Some of these so-called superheavy elements of atomic numbers $Z>103$ have been detected only in the form of extremely short-lived atomic nuclei, whereas atoms with their shells of electrons have escaped detection. Nonetheless, they are recognized as proper elements no less real than oxygen and iron. To Hendry, this not only raise questions regarding criteria of existence, it also relates to the problem of the existence of composite bodies in general or what in philosophical circles is known as the "Special Composition Question.”

As stated by the editors of What Is a Chemical Element? the aim of the book is "to provide an update to the current state of the debate on elements" (p. 2). The book is more than just an update, though, as it offers a series of wide-ranging and in part innovative scholarly analyses of the subject. The attentive reader will not find a final answer to what an element is, but he or she will better appreciate the complexity and many facets of the question.
The book is written by specialists in the philosophy of chemistry and mainly addressed to other specialists, and for this reason it is less relevant to the average chemical reader with an interest in the foundational problems of chemistry. For better or for worse, it illustrates how philosophy of chemistry has come of age as an independent branch of academic philosophy of science. With the independence follows almost inevitably a specialized academic language and a scholarly style that makes the new research area uninviting or even slightly incomprehensible to outsiders. In my opinion, the primary audience of What Is a Chemical Element? will primarily be philosophers of science and secondarily historians of chemistry. 